\title{
Atomistic Simulations of Mechanics of Nanostructures
}

\section{Hanchen Huang and Helena Van Swygenhoven, Guest Editors}

\begin{abstract}
Nanostructures can be in the form of nanoparticles or nanograins, nanowires or nanotubes, and nanoplates or multilayers. These nanostructures may be used individually or embedded in a bulk material. In both cases, they share two common features. First, the small dimensions minimize or even eliminate the presence of defects. Second, nanostructures entail large surface or interface areas. The absence of defects makes nanostructure materials stronger than their bulk counterparts, leading to the eventual realization of ideal strength. The presence of surfaces and interfaces may either reduce or increase the strength. Atomistic simulations can provide insight into the deformation mechanism at the atomic and electronic level, something that is very difficult to obtain from experiments. This article describes generic features of nanostructures and summarizes the five areas presented in the articles in this issue.
\end{abstract}

\section{Introduction}

A structure becomes nanoscale when at least one of its three dimensions is below $100 \mathrm{~nm}$. Gleiter ${ }^{1}$ classified nanostructures according to their dimensionality, as schematically shown in Figure 1a. In nanoparticles or nanograins, all three dimensions are nano, making them zerodimensional (0D). Nanowires or nanotubes are large in only one dimension, making them one-dimensional (1D). Analogously, thin films, multilayers, or membranes exceed the nanometer regime in two of the three dimensions, making them two-dimensional (2D). Confinements of electron distribution in small dimensions can lead to both quantitative and qualitative changes of materials functionalities. In connection with mechanics, these three types of nanostructures share two common features. On one hand, the smaller the structure, the less chance it incorporates defects. On the other hand, the number of atoms situated in the surface or interface increases drastically. These two structural characteristics of nanostructures determine, to a great extent, their elastic and plastic responses in the presence of surfaces and interfaces. For example, the Young's modulus of a nano- structure can increase or decrease with dimension, as shown in Figure 1b. These aspects form the focus of this theme issue.
At an interface, the periodicity of atomic arrangement terminates. This termination means the loss of atomic neighbors at a free surface, and therefore the change of electron distribution. As an example, Figure 2 shows electron redistribution near a $\mathrm{ZnO}$ (1120) surface. ${ }^{2}$ Due to the loss of atomic neighbors, each of the surface bonds (S1, S2, and S3) is strengthened, as indicated by the overlap of electron distribution (as shown by the red contours in Figure 2). This tendency of electron redistribution moves the surface atoms and exerts strain on atoms under the surface. At a metallic surface, similar electron redistribution occurs, leading to surface stress and strain. The surface stress may lead to or facilitate surface reconstruction, change of elastic moduli, phase transformation, dislocation nucleation and motion, and self-organization of nanostructures. ${ }^{3-5}$ When surface stress itself is insufficient, additional mechanical stress helps facilitate the phase transformation. 5,6,7

Local electronic structure plays a key role in determining the nature of defects in crystals. For instance, the general planar fault energy curves, which represent the energy dependency of rigidly shearing a crystal, influence the nature of slip activity in nanocrystalline systems. ${ }^{8}$ Figure 3a displays the valence electron density in face-centered-cubic (fcc) Al that is rigidly sheared at a $\{111\}$ plane along the $\langle\overline{11} 2\rangle$ slip direction. The inter $\{111\}$-plane bonding is substantially altered. This alteration results in the maximum energy configuration in the generalized stacking fault energy (GSFE) curve displayed in Figure

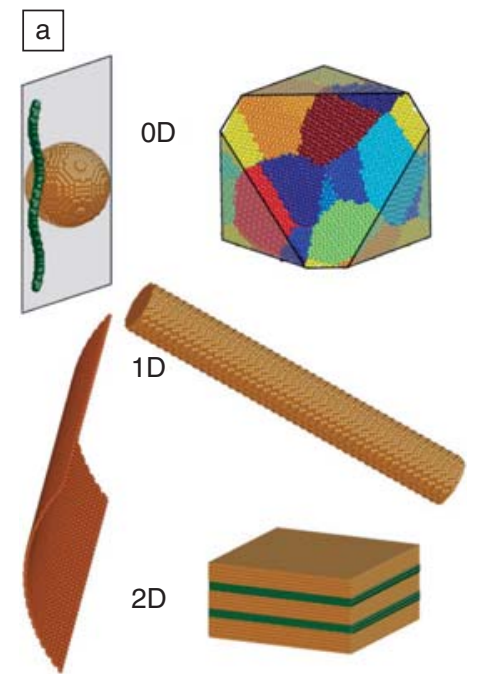

$\mathrm{b}$

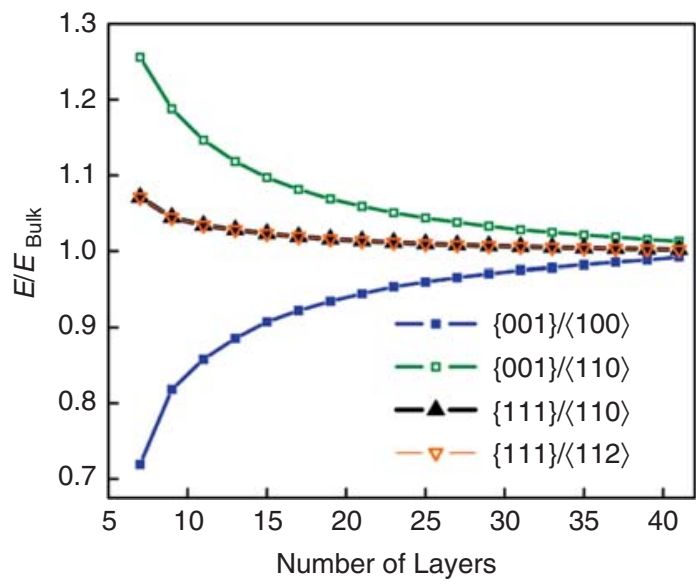

Figure 1. (a) Schematic of nanostructures-OD nanoparticles and nanograins, 1D nanowires, and 2D membranes and multilayers; (b) Variation of normalized Young's modulus $(E)$ as a function of $\mathrm{Cu}$ nanoplate thickness. Reprinted with permission from Reference 3. () 2004, American Institute of Physics. 
$3 \mathrm{~b}$ and is referred to as the unstable stacking fault energy. ${ }^{9}$ Atomistic simulations have shown that when the unstable stacking fault energy is close in value to the stable stacking fault energy (such as in $\mathrm{Al})$, the nucleation of full dislocations at the grain boundary becomes more likely within the simulation time. ${ }^{8}$ A similar rationale applies to twin fault and twin migration activity within the nanocrystalline system. ${ }^{10}$ Beyond the demonstrating examples in Figures 2 and 3, interfaces serve as sinks and sources of defects and conspire with dislocations in a variety of mechanical deformation processes.

Atomistic simulations have been instrumental to the understanding of the deformation mechanism responsible for the mechanical behavior of materials and are particularly prominent for nanostruc-

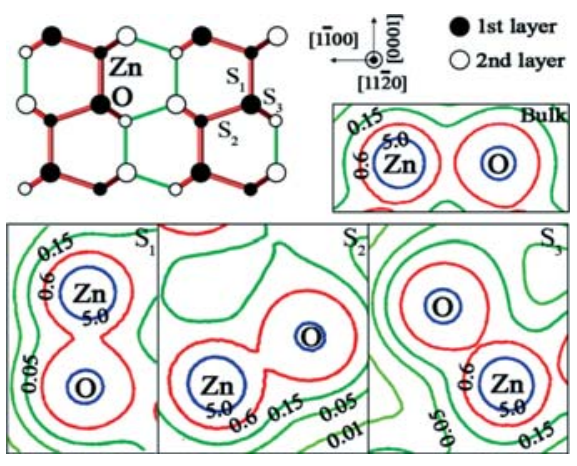

Figure 2. Top view of the atomic structure of the $\mathrm{ZnO}(11 \overline{2} 0)$ surface, the density contour of electrons at $S_{1}, S_{2}$, and $S_{3}$, and that in the bulk for comparison. Reprinted with permission from Reference 2. (C) 2006, American Institute of Physics. tures. Individual nanostructures are generally much smaller than $100 \mathrm{~nm}$ in dimension. This dimension is well within the reach of classical atomic simulations such as molecular dynamics and kinetic Monte Carlo simulations. For those of even smaller dimensions, electronic (full electronic or tight-binding) calculations are feasible, providing full details of atomic/electronic behavior. Such details are usually beyond the reach of direct experimental characterizations, and yet they dictate the mechanical deformation of nanostructures.

In synergy with experiments, the atomistic simulations can provide useful guidance to experiments in suggesting deformation mechanisms, especially since the method provides direct access to many "hidden" parameters in experiments such as the interface structure at the atomic and electronic level, the stored excess energy, the free volume, and the internal stress distribution. ${ }^{11}$ However, atomistic simulations, even at the full electronic level, involve approximations and idealizations such as the representation of many-body electronic effects. The short time steps required in molecular dynamics result in using strain rates that are incredibly high when compared with experimental values. Therefore, extrapolation toward experimental observations has to be done with extreme care, making it almost impossible to determine the true rate-limiting processes.

There are, however, several success stories of synergetic effects between simulations and experiments such as surface reconstruction and the dislocation mechanism in nanograins, both of which have been predicted by atomistic simulations and confirmed by experiments.
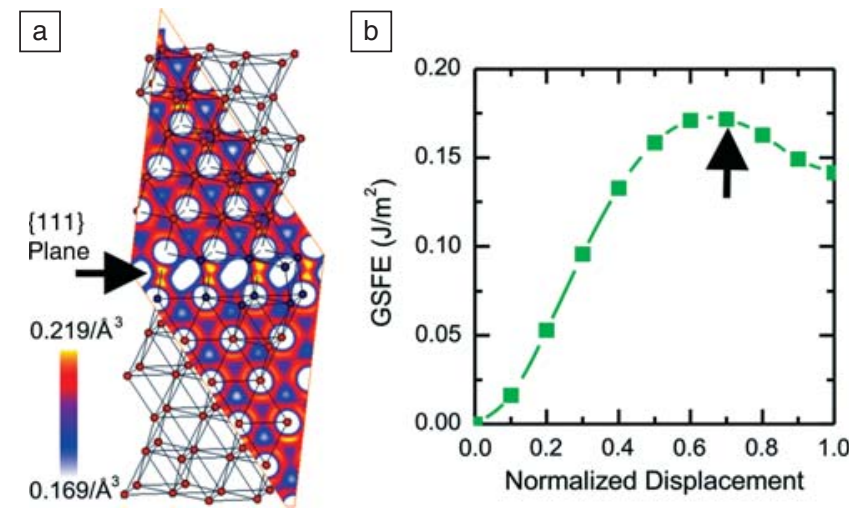

Figure 3. Al fcc (face-centered cubic) electronic charge density map of a $\{111\}$ plane that intersects a rigidly sheared $\{111\}$ plane along the $\langle\overline{1} 12\rangle$ slip direction. (b) The energy dependence as a function of normalized rigid displacement-the generalized stacking fault energy (GSFE) curve. In (a) the configuration is taken at the maximum energy of (b) (black arrow), the unstable stacking fault energy.

\section{Coverage of This Issue}

This issue focuses on crystalline nanostructures. Nanotubes are not the main focus because they have been covered in previous issues of the MRS Bulletin. ${ }^{12}$ Two aspects of mechanics are addressed by simulations: mechanical deformation driven by stress or strain and mechanics-driven synthesis of nanostructures. The five overview articles cover the topics of ultra-strength of nanostructures, the strengthening role of nanoparticles, elastic and plastic responses of nanowires, plastic deformation of nanoscale multilayers and nanograins, 13,14 and advancements in mechanics-driven synthesis of nanostructures.

Looking at a generic feature, Zhu et al. review recent advancements in understanding the ultra-high strength of nanostructures. The strength is dictated by dislocation nucleation and motion at low temperatures. In the absence of mobile dislocations, plastic deformation will not proceed until new dislocations are nucleated, which can lead to a much higher strength, approaching the ideal strength. The ultra-high strength is reachable, for example, during nanoindentation of single crystalline solids. When dislocations, although available to facilitate plastic deformation, do not have sufficient time to respond to a stress, the strength can also increase; this is the case in high strain-rate deformation. In nanostructures, additional obstacles such as interfaces and nanoparticles also serve to increase the strength. Zhu et al. review the length- and time-scale effects on the deformation processes in nanostructures with ultrahigh strength. They emphasize the critical role of activation volume in understanding the strength-controlling mechanisms.

One possible application of the extremely high strengths of nanoparticles is to exploit their role as reinforcement to improve the mechanical properties of bulk materials. Chrzan et al. consider the strengthening of alloys through the introduction of nanoscale precipitates, hindering the propagation of dislocations in the crystalline matrix. The precise mechanism by which nanoparticles strengthen a bulk material is size dependent: The very small particles can be cut by dislocations, whereas dislocations are forced to circumvent larger precipitates. The structure of the precipitates also can be altered through its interaction with dislocations. However, exceptional structural stability and strength can be obtained by introducing nanoscale particles; for example, small nanoparticles of 3-5 $\mathrm{nm}$ in diameter can be structurally stable at high temperatures and lead to a six orders-of-magnitude increase in creep resistance. The deforma- 
tion mechanisms derived from atomistic simulations are critical input for elasticitybased dislocation dynamics, a simulation technique at a higher lengthscale. ${ }^{15-17}$

Focusing on 1D nanostructures, Park et al. review recent developments in nanowire mechanics. Near surfaces, atoms have fewer bonding neighbors, leading to a redistribution of electrons that results in the rearrangement of surface atoms for metallic solids or reconstructions for covalent solids. The rearrangement or reconstruction also leads to elastic stiffening or softening of the surfaces. At the same time, it also imposes a large strain on the nanowire interior, triggering nonlinear elastic stiffening or softening. When the cross-sectional dimension is small enough, surface rearrangement or reconstruction also can lead to phase transformations, such as fcc to body-centered-tetragonal (bct) in gold nanowires. Defects nucleated due to surface stresses can couple with those created due to externally applied forces. Under large deformation, the strength of nanowires depends on whether nucleation or glide requires larger stress. As a result, metallic nanowires can become either stronger or slightly weaker with the introduction of twin boundaries.

Focusing on 2D multilayers and nanocrystalline metals where each crystal is a nanograin, Derlet et al. review recent advancements in mechanics in confined volumes, particularly the interaction between dislocations and grain boundaries. The complex lifetime of a dislocation is reviewed, starting with its nucleation at the grain boundary, followed by its propagation and eventual absorption in the surrounding grain boundary network. Dislocation nucleation at grain boundaries is studied in systematic bicrystal simulations that consider coincident site lattice boundaries and in simulations using fully 3D crystalline networks in which general high angle boundaries with tilt and twist components can be studied. The latter also allows the study of the effect of triple and higher order junctions on the dislocationinterface interactions. The unique properties of fcc/bcc (body-centered cubic) nano-layered composite systems and the details of their interface properties are also reviewed. Their concluding discussion focuses on the limitations of finite temperature molecular dynamics. New transition path methods may be used to overcome the inherent high-strain rate/high-stress regime through the calculation of activation volumes that characterize the strainrate sensitivity of the atomic scale plastic processes studied.

Turning to the applications of nanomechanics, Liu et al. review recent advancements in mechanics-driven synthesis of nanostructures. Strain generates long-range fields, such as Columbic fields, of charged particles. This long range nature enables the self-organization of structures so as to minimize the thermodynamic free energy. Quantum dot formation during hetero-epitaxy is one such example. Driven by the mismatch strain between epilayer and substrate, the quantum dots tend to be periodic and uniform to minimize the strain energy. Size uniformity in the synthesis of these quantum dots affects their functional behavior, such as monochromic light emission. Crystalline surfaces, already under intrinsic surface stress, are very responsive to additional stresses. Introduction of gas molecules onto a surface can generate sufficient stress to peel off the surface layer and drive it to form tubular structures. This mechanics-driven selforganization of tubular nanostructures offers the advantage of structure control. In contrast, vapor-based synthesis usually leads to nanostructures of mixed structures (e.g., nanotubes of mixed chirality).

\section{Prospects}

The overview articles in this volume show that atomistic simulations have had a large effect on the advancement of nanostructure mechanics, and there is still opportunity for further advances. Much effort has been invested in the basic science of nanostructure mechanics. ${ }^{18}$ Looking forward, there are two areas that pose great challenges and offer potentials of reward.

The first area is the bridging of time scales in atomistic simulations. In contrast to the bridging of length scales, there has been less progress in bridging time scales. Both electronic structure and classical molecular dynamics simulations are limited to nanoseconds in time scale. Hyper-molecular dynamics and similar approaches enable simulations lasting microseconds for selective materials systems in which diffusion kinetics is simpler. Unfortunately, this condition is not satisfied most of the time. By associating atoms to lattice points, lattice kinetic Monte Carlo simulations extend the time scale to microseconds or seconds. However, the use of the lattice renders it impossible to directly represent mechanical deformation. Fortunately, strain and stress can be represented by strain energy. Incorporating strain energy into the lattice kinetic Monte Carlo simulations and coupling this method with other simulation methods (such as classical molecular dynamics and a continuum approach) may lead to atomistic methods over multiple time scales.

The second area concerns the hierarchy of nanostructures. So far, the majority of atomistic simulations have focused on a single type of nanostructure. Realistic applications will likely involve hierarchies of nanostructures. For example, a future integrated circuit may include doped nanowires or nanotubes as $p-n$ junctions, nanofilms as insulators, and nanoparticlereinforced composites as case materials. The hierarchies will likely include heterointerfaces where charge transfer is critical. Coupling of electronic structure calculations and classical molecular dynamics simulations may enable rigorous simulations of charge transfer and also allow the efficient treatment of large dimensions.

\section{Acknowledgments}

$\mathrm{HH}$ gratefully acknowledges the financial support from the U.S. National Science Foundation (NSF) (CMMI 0625602, 0553300, and 0727413) and the Department of Energy Office of Basic Energy Sciences (DE-FG02-04ER46167). Thanks to Dr. Ken Chong for his visionary leadership in nanomechanics research and well wishes as he retires from NSF. HVS greatly acknowledges that financial support of the Swiss National Science Foundation and the European Commission (FP6NANOMESO, Grant No. 016710).

\section{References}

1. H. Gleiter, Acta Mater. 48, 1 (2000).

2. L.X. Zhang, H.C. Huang, Appl. Phys. Lett. 89, 183111 (2006).

3. L.G. Zhou, H.C. Huang, Appl. Phys. Lett. 84, 1940 (2004)

4. Y.F. Zhang, H.C. Huang, Nanoscale Res. Lett. 4, 34 (2009).

5. L.X. Zhang, H.C. Huang, Appl. Phys. Lett. 90, 23115 (2007)

6. C.L. Freeman, F. Claeyssens, N.L. Allan, and J.H. Harding, Phys. Rev. Lett. 96, 066102 (2006).

7. P.W. Chung, Phys. Rev. B 73, 75433 (2006)

8. H. Van Swygenhoven, P.M. Derlet, A.G. Froseth, Nat. Mater. 3, 399 (2004).

9. C. Brandl, P.M. Derlet, H. Van Swygenhoven, Phys. Rev. B 76, 054124 (2007).

10. A.G. Frøseth, H. Van Swygenhoven, P.M. Derlet, Acta Mater. 52, 2259 (2004).

11. H. Van Swygenhoven, J.R. Weertman, Mater. Today 9, 24 (2006).

12. M.S. Dresselhaus, H. Dai, Eds., MRS Bull. 29 (4) (2004).

13. R.P. Vinci, S.P. Baker, Eds., MRS Bull. 27 (1) (2002).

14. H. Kung, T. Foecke, Eds., MRS Bull. 24 (2) (1999).

15. N.M. Ghoniem, S.H. Tong, L.Z. Sun, Phys. Rev. B 61, 913 (2000)

16. M. Rhee, H.M. Zbib, J.P. Hirth, H. Huang, T. de la Rubia, Modell. Simul. Mater. Sci. Eng. 6, 467 (1998).

17. B. Devincre, T. Hoc, L. Kubin, Science 320, 1745 (2008).

18. K.P. Chong, J. Phys. Chem. Solids 65, 1501 (2004). 


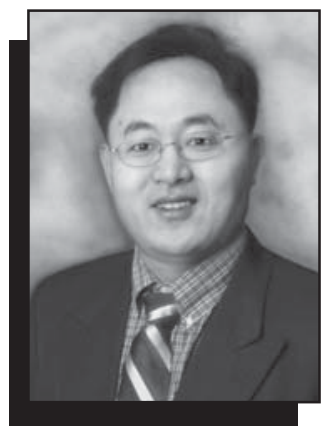

Hanchen Huang

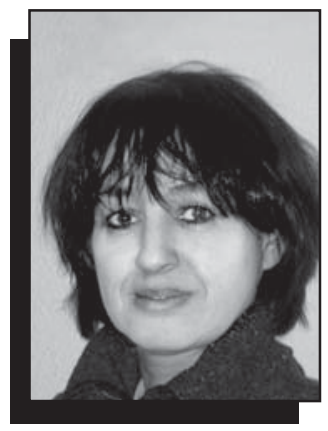

Helena Van Swygenhoven

by complementary experiments.

Editor for this issue of MRS Bulletin, can be reached at the Department of Mechanical, Aerospace, and Nuclear Engineering, Rensselaer Polytechnic Institute, and the Department of Mechanical Engineering, University of Connecticut; tel. 518276-2020; e-mail hanchen@rpi.edu or hanchen@engr.uconn. edu.

Huang is a professor of mechanical and nuclear engineering at Rensselaer Polytechnic Institute and is in transition to be the SoE Professor of Sustainable Energy at the University of Connecticut. Before this appointment, he taught at Hong Kong Polytechnic University and worked at Lawrence Livermore National Laboratory. Huang has an interdisciplinary background, with a BS degree in physics, MS degree in theoretical nuclear physics, $\mathrm{PhD}$ degree in nuclear engineering, postdoctorate training in materials/mechanics modeling, and teaching experience in mechanical and nuclear engineering. His primary research area is nanosynthesis on surfaces and spans over mechanics and radiation damage near surfaces and interfaces. His primary expertise is in atomistic simulations, augmented

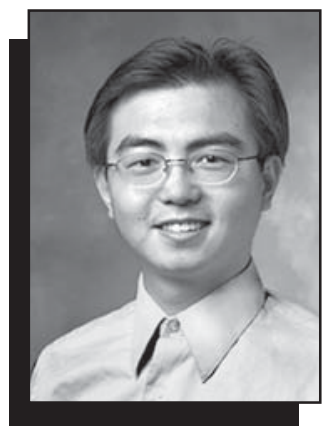

Wei Cai

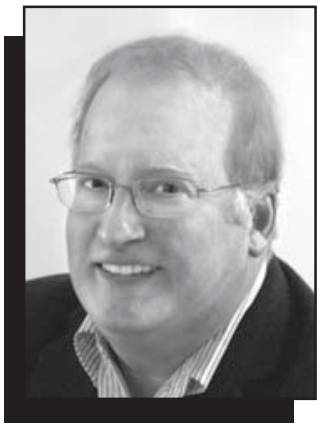

Daryl C. Chrzan

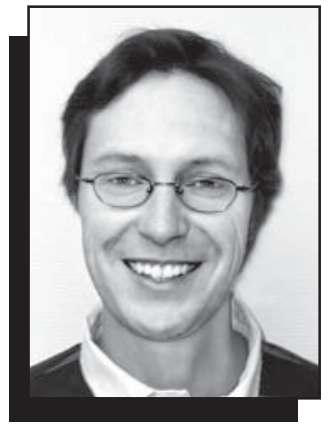

Peter M. Derlet
National Science Foundation, European Framework programs, and Swiss Competence Center for Material Science and Technology. She has co-organized several international conferences, workshops, and MRS symposia to promote a synergy between modeling, theory, and experiment, has served as a volume organizer in 2004 for MRS Bulletin, and chaired the 2006 MRS Fall Meeting.

Wei Cai can be reached at the Department of Mechanical Engineering, Stanford University, 496 Lomita Mall, Durand 211, Stanford, CA 943054040, USA; tel. 650-7361671, fax 650-725-1587, and e-mail caiwei@ stanford.edu.

Cai is an assistant professor of mechanical engineering at Stanford University. Before this appointment, he was a Lawrence Postdoctoral Fellow at Lawrence Livermore National Laboratory. Cai received his BS degree in optoelectronic engineering from Huazhong University of Science and Technology, China, and his $\mathrm{PhD}$ degree in nuclear engineering from the Massachusetts Institute of Technology. His primary research area is the fundamental mechanisms of inelastic deformation of crystalline materials at the micro- and nanoscales. His primary expertise is in atomistic and dislocation dynamics simulations.

\section{Daryl C. Chrzan can be} reached at the

Department

of Materials Science and Engineering at the University of California, Berkeley, CA 94720,

USA; tel. 510-643-1624, and e-mail dcchrzan@ berkeley.edu.

Chrzan is a professor of materials science at the University of California, Berkeley. He received his $\mathrm{PhD}$ in condensed matter physics from the University of California, Berkeley. He was a senior member of the technical staff at Sandia National Laboratories from 1990-1995. In 1995, he joined the Materials Science and Engineering Department at the University of California, Berkeley. In addition, Chrzan is currently a faculty staff scientist at the Lawrence Berkeley National Laboratory.

Peter M. Derlet can be reached at the Materials Science and Simulation division of the Paul Scherrer Institute, Switzerland; tel. 41-56310-3164, fax 41-56-3103131, and e-mail peter.derlet@psi.ch.
Derlet is a senior staff scientist at the Paul Scherrer Institute, Switzerland. He received his PhD in theoretical particle physics from Monash University, Australia, in 1995. Since then, he has undertaken post-doctoral research in Australia and Norway in the fields of condensed matter and materials science, with an emphasis on materials modeling. His current research interests primarily lie in the investigation of the dynamical, structural, and mechanical properties of novel metallic materials using atomistic simulation methods.

Horacio D. Espinosa can be reached at the Department of Mechanical Engineering at Northwestern University, 2145 Sheridan Road, Evanston, IL 60208-3111, USA; tel. 847-467-7673, fax 847-491-3915, and e-mail espinosa@north western.edu.

Espinosa is a professor of mechanical engineering at the McCormick School of Engineering at Northwestern University. Espinosa received his $\mathrm{PhD}$ in applied mechanics at Brown University in 1992. He has made contributions in the areas of dynamic failure of advanced materials, micromechanics, and 


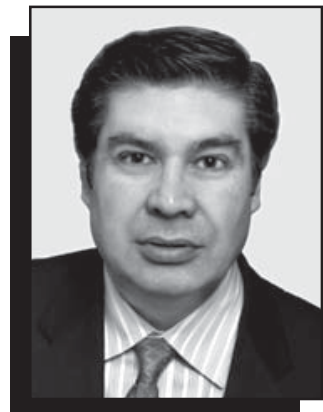

Horacio D. Espinosa

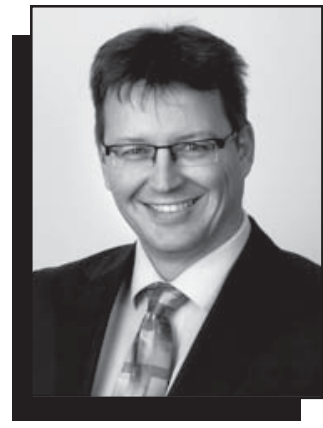

Peter Gumbsch

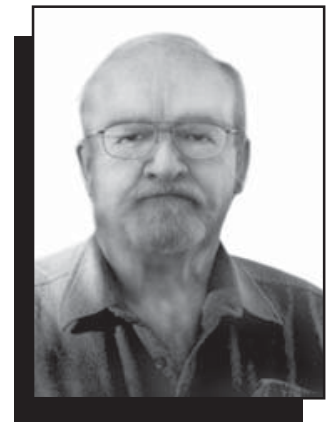

Richard G. Hoagland

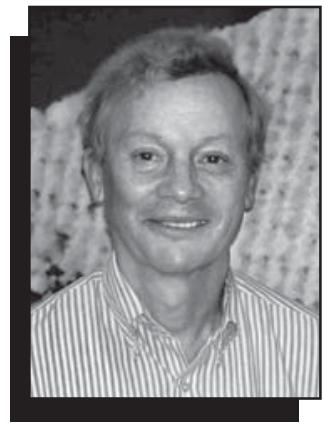

Max G. Lagally

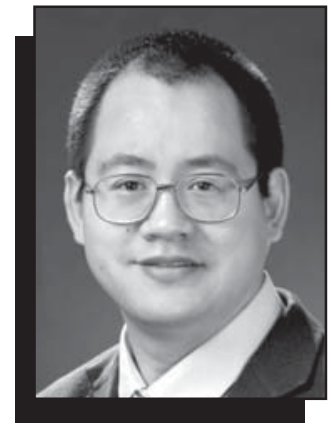

Ju Li nanomechanics. He has published more than 150 technical papers in these fields. Espinosa has received two Young Investigator Awards, NSF-Career and ONRYIP awards, the American Academy of Mechanics (AAM) 2002Junior Award, the Society for Experimental Mechanics (SEM) 2005 HETENYI Award, the Society of Engineering Science 2007 Junior Medal, and the 2007 LAZAN award from SEM. He is a fellow of AAM, ASME, and SEM. He currently serves as editor-in-chief of the Journal of Experimental Mechanics, co-editor of the Wiley book series on micro and nanotechnologies, and as an associate editor of the Journal of Applied Mechanics. His current research interests are on size scale plasticity and fracture of nanostructures, microelectromechanical systems, nanoelectromechanical stystems, in situ electron and atomic probe microscopy testing of nanostructures, and the development of microdevices for massively parallel atomic probe nanofabrication of the next generation of electronic circuits and biochips.

Peter Gumbsch can be reached by e-mail at peter.gumbsch@iwm.

fraunhofer.de.

Gumbsch is a professor for mechanics of materials at the

University of Karlsruhe (TH) and head of the Fraunhofer Institute for Mechanics of Materials IWM in Freiburg and Halle. His research activities focus on modeling and simulation of materials using atomistic simulation, mesoscopic modeling, as well as macroscopic materials descriptions. Central research topics are deformation and fracture processes as well as interface properties in metals and ceramics.

Gumbsch has authored more than 120 refereed journal publications and has been awarded the Otto Hahn Medal (1992), the Masing Memorial Award (1998), and the Gottfried Wilhelm Leibniz Prize (2007).

\section{Richard G. Hoagland} can be reached at the Los Alamos National Laboratory, Los Alamos, NM 87545, USA; tel. 505667-3098, fax 505-667-

8021, and e-mail hoagland@lanl.gov.

Hoagland is a technical staff member in the Materials Science and Technology Division at Los Alamos National Laboratory. He is professor emeritus of Washington State
University and a Fellow of the American Society of Metals. He received his PhD degree in metallurgical engineering from The Ohio State University in 1973. He has approximately 150 publications in books and journals. His work focuses on modeling structure-property relations.

Max G. Lagally can be reached at the Department of Materials Science and Engineering, University of Wisconsin-Madison, 1509 University Ave., Madison, WI 53706, USA; tel. 608-263-2078, fax 608-265-4118, and e-mail lagally@engr. wisc.edu.

Lagally is the Erwin W. Mueller Professor of Materials Science and Physics at the University of Wisconsin-Madison. His research focuses on growth and structure-property relationships of materials at the nanoscale, primarily semiconductors. He has more than 350 publications, has edited four books, and holds 10 patents. He is a member of the U.S. National Academy of Engineering and the German National Academy of Sciences Leopoldina and has received numerous other awards, including the
MRS Medal. He is the founder of two companies, nPoint Inc. and SonoPlot Inc., both based on technology developed in his labs.

$\mathrm{Ju} \mathbf{L i}$ can be reached at the Department of Materials Science and Engineering at the University of Pennsylvania, 223 LRSM, 3231 Walnut Street, Philadelphia, PA 19104, USA; tel. 215-898-1558, fax 215-573-2128, and e-mail liju@seas.upenn.edu.

$\mathrm{Li}$ is an associate professor of materials science and engineering at the University of Pennsylvania. The Li group (http: / / mt.seas. upenn.edu) performs theoretical and computational research on mechanical properties of nanostructured materials, cell mechanics, transport, energy storage and conversion, catalysis, and metamaterials. Li earned his $\mathrm{PhD}$ degree in nuclear engineering from MIT in 2000. He has published more than 80 peerreviewed papers and is the recipient of the 2005 Presidential Early Career Award for Scientists and Engineers, the 2006 MRS Outstanding Young Investigator Award, and the 2007 TR35 award from Technology Review.

Feng Liu can be reached at the Department of
Materials Science and Engineering at the University of Utah, Salt Lake City, Utah, 84112 , USA; tel. 801-587-7719, and e-mail

fliu@eng.utah.edu.

Liu is a professor in the Department of Materials Science and Engineering at the University of Utah. His research interests lie in materials modeling and simulation from the atomic to mesoscopic scales, with a recent focus on properties of surfaces, interfaces and nanostructures, mechanisms of growth of thin films and of selfassembly of nanostructures, and nanomechanical architectures of nanomembranes. Liu received his $\mathrm{PhD}$ degree in chemical physics from Virginia Commonwealth University and was a postdoctoral researcher at Rutgers University and Oak Ridge National $\mathrm{Lab}$. He is a member of the American Physical Society and Materials Research Society. He has authored 130 publications and holds four patents. He received the Humboldt Senior Research Award in 2008.

David L. McDowell can be reached at the School of Materials Science and Engineering at the Georgia Institute of 


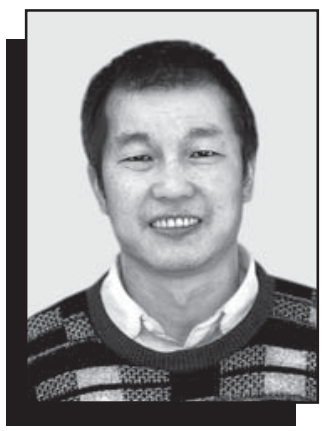

Feng Liu

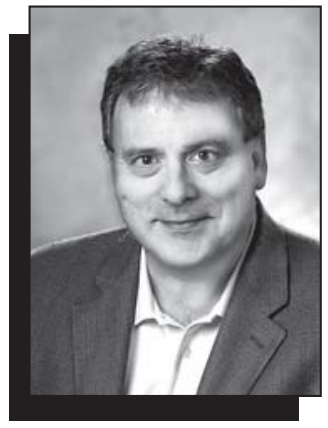

David L. McDowell

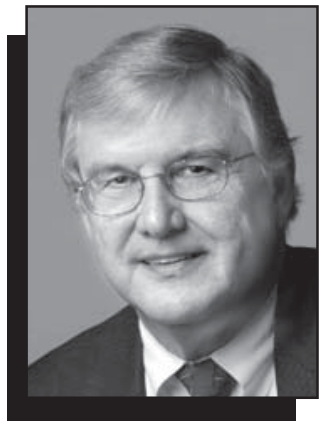

John William Morris, Jr.

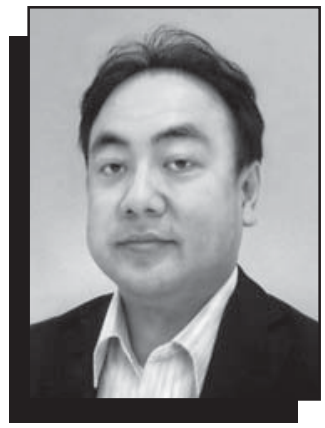

Shigenobu Ogata

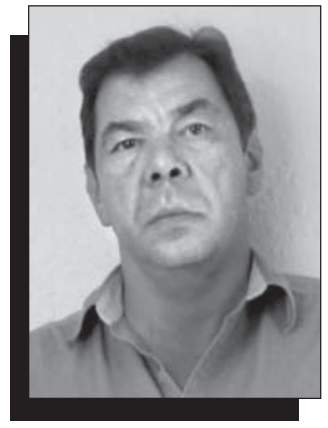

Yuri Osetsky
Technology, Atlanta, GA 30332-0245, USA; tel. 404-894-5128,

fax 404-894-0186, and e-mail david.mcdowell@ me.gatech.edu.

McDowell is the Regents' Professor and Carter N. Paden, Jr. Distinguished Chair in Metals Processing at Georgia Tech. He received a $\mathrm{PhD}$ degree from the University of Illinois in 1983 and joined Georgia Tech thereafter. McDowell actively works in atomistic modeling of dislocations, constitutive models for cyclic plastic deformation and fatigue, multiscale kinematics and kinetics of plastic deformation in polycrystals, hierarchical multiscale behavior of heterogeneous materials, and materials design. He is the author or co-author of more than 300 publications and has received the ASME Materials Division Nadai Award (1997) and the 2008 Khan International Medal for outstanding contributions to the field of metal plasticity.

John William Morris, Jr. can be reached at the Department of Materials Science and Engineering at the University of California, Berkeley, CA 94720, USA; e-mail jwmorris@berkeley.edu.

Morris is a professor of metallurgy at the
University of California, Berkeley. He received a ScD from MIT in 1969 and joined the Berkeley faculty in 1971. His research has focused on mechanical properties of metals and alloys, including structural steels, aluminum, and solder materials. He is a member of the National Academy of Engineering and a fellow of the Materials Research Society (MRS), the Metallurgical Society (TMS), and ASM International. He also is a recipient of Berkeley's Distinguished Teaching Award.

Shigenobu Ogata can be reached at the Department of Mechanical Science and Bioengineering at Osaka University, Osaka

560-8531, Japan; e-mail: ogata@me.es. osaka-u. ac.jp.

Ogata is a professor of mechanical science and bioengineering at Osaka University, Japan. His research interests include the first principles modeling of materials, the modeling of nonlinear and nonequilibrium behaviors of materials, and understanding materials deformation mechanisms. Ogata received his PhD degree in mechanical engineering from the Osaka University (1998) and has been a visiting scientist at the Massachusetts Institute of Technology (2001-2002).

Yuri Osetsky (aka Yury Osetskiy) can be reached at the Oak Ridge National Laboratory, Oak Ridge, TN 37831-6138, USA; tel. 865-576-3254, fax 865-241-3650, e-mail osetskiyyn@ornl.gov.

Osetsky has received BSc and MSc degrees in physics from the Moscow Engineering Physics Institute and $\mathrm{ahD}$ in physics and mathematics from the Russian Research Centre

"Kurchatov Institute." He has worked at the Atomic Energy Institute

(Moscow, Russia), CIEMAT (Madrid, Spain), UPC (Barcelona, Spain), and Liverpool University (UK). Osetsky currently is a senior staff member at the Materials Science and Technology Division at Oak Ridge National Laboratory in Tennessee. Fields of interest include the radiation damage of metals, atomic-scale dislocation dynamics, and multiscale study of materials properties.

Harold S. Park can be reached at the Department of Mechanical Engineering at the University of Colorado, Boulder, CO 80309, USA; tel. 303-492-7750, fax 303-492-3498, and e-mail harold.park@ colorado.edu.

Park is an assistant professor of mechanical engineering at the University of Colorado. He received his BS, MS, and $\mathrm{PhD}$ degrees in mechanical engineering from Northwestern University. During his PhD work, he developed novel multiscale simulation techniques that coupled atomistics to continua. At Colorado (http://cumcen.org/ haroldp/), his research centers on using both atomistic and multiscale simulation techniques to elucidate surface effects on the mechanical behavior and properties of metallic and semiconducting nanowires. He is the recipient of the 2007 National Science Foundation CAREER award and a 2008 DARPA Young Faculty Award.

Roger Stoller can be reached at the Oak Ridge National Laboratory, Oak Ridge, TN 37831-6138, USA; tel. 865-576-7886, fax 865-241-3650, and e-mail: stollerre@ornl.gov.

Stoller is a distinguished research staff member and fusion materials program manager at the Materials Science and Technology Division of the Oak Ridge National Laboratory. He received

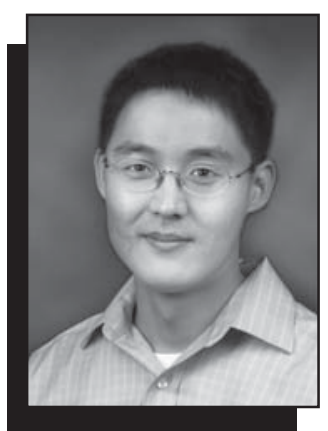

Harold S. Park

a BS in nuclear engineering from the University of California, Santa Barbara (UCSB) in 1978, a MS in nuclear engineering from the University of Wisconsin-Madison in 1979, and a PhD in chemical engineering from UCSB in 1987. His primary research interest is computational modeling and simulation of radiation effects in structural materials. He is the author or co-author of more than 100 publications, a fellow of ASM International and ASTM International, and currently serves as vice chairman on the ASTM Board of Directors.

Jian Wang can be reached at the Los Alamos National Laboratory in Los Alamos, NM 87545, USA; tel. 505-667-1238, fax 505-667-8021, and e-mail wangj6@lanl.gov. 


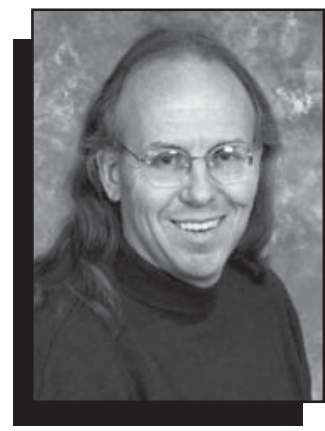

Roger Stoller

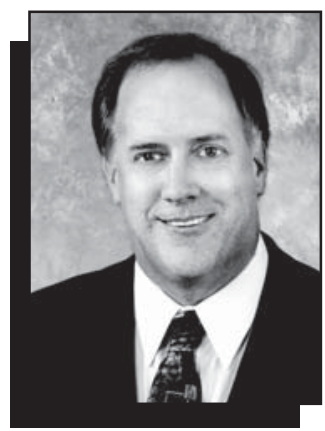

Steven J. Zinkle

Wang is a postdoctoral research associate in the Materials Science and Technology Division at Los Alamos National Laboratory. He received his $\mathrm{PhD}$ degree in mechanical engineering from Rensselaer Polytechnic Institute in 2006. He has approximately 45 publications in books and journals. His work focuses on modeling deformation behavior of composites and growth mechanisms of nanostructures.

Sidney Yip can be reached at the Department of Nuclear Science and Engineering at the Massachusetts Institute of Technology,

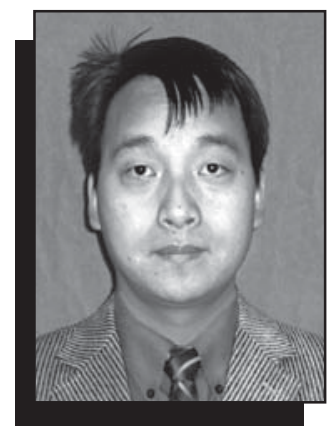

Jian Wang

Cambridge, MA 02139,

USA; e-mail

syip@mit.edu.

Yip has been a professor of nuclear science and engineering since 1965 and a professor of materials science and engineering since 2000 at the

Massachusetts Institute of Technology. After receiving undergraduate and graduate degrees from the University of Michigan, he spent a year as a postdoctoral fellow at the University of Michigan and two years as a research associate at Cornell University. His interest lies in multiscale materials modeling theory and simulation, particularly at the atomistic level. Most recently he edited the Handbook of Materials Modeling (Springer, 2005). He is a fellow of the American Physical Society and a member of the Materials Research Society.

Ji Zang can be reached at the School of Chemical and Biomolecular

Engineering at the Georgia Institute of

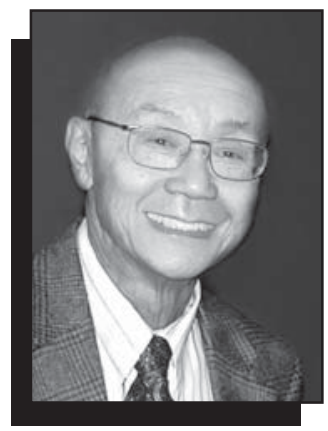

Sidney Yip

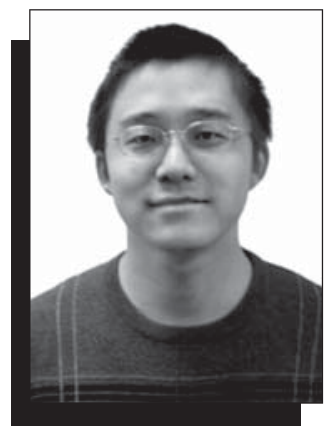

Ji Zang

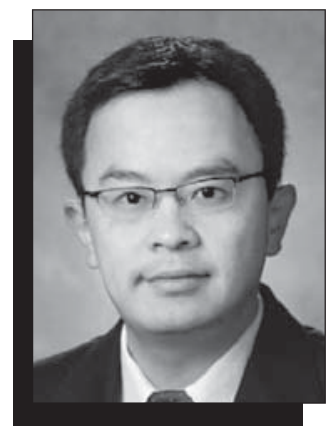

Ting Zhu
Technology, Atlanta, GA 30332, USA; tel. 404-894-7608, and e-mail ji.zang@chbe. gatech.edu.

Zang is a postdoctoral fellow at the Georgia Institute of Technology and works on the diffusion of water and gas molecules in carbon nanotubes and metal oxide nanotubes. He received his BS and MS degrees from the School of Materials Science and Engineering at $\mathrm{Xi}^{\prime}$ an Jiaotong University, Xi'an, China, in 1999 and 2001, respectively. In 2007, Zang obtained his PhD degree in materials science and engineering at the University of Utah, where he focused on theoretical modeling and molecular

dynamics simulation of mechanical properties of carbon nanotubes under hydrostatic pressure and bending behavior of $\mathrm{Si} / \mathrm{Ge}$ nanofilms.

Ting Zhu can be reached at the Woodruff School of Mechanical Engineering at the
Georgia Institute of Technology, Atlanta, GA 30332, USA; e-mail ting.zhu@me.gatech.edu. Zhu is an assistant professor in the Woodruff School of Mechanical Engineering at the Georgia Institute of Technology. His research is focused on materials modeling and nanomechanics of materials (http://www.me.gatech. edu/zhugroup/). Zhu received his $\mathrm{PhD}$ degree from the Massachusetts Institute of Technology (2004) and was a postdoctoral research associate at Harvard University (2004-2005).

Steven J. Zinkle can be reached at the Materials Science and Technology Division, Oak Ridge National Laboratory (ORNL), P.O. Box 2008, Oak Ridge, TN 378316132, USA; tel. 865-5744065, fax 865-574-4066, and e-mail zinklesj@ornl.gov.

Zinkle is a corporate fellow and director of the Materials Science and Technology Division at ORNL. He received a $\mathrm{PhD}$ in nuclear engineer- ing and an MS in materials science from the

University of

Wisconsin-Madison in 1985 and subsequently joined ORNL as a Wigner Fellow. He has been a visiting scientist at Risø National Laboratory in Roskilde, Denmark and Forschungszentrum Jülich in Germany. His research interests include deformation and fracture mechanisms in structural materials and investigation of radiation effects in metals and ceramics for fusion and fission energy systems. He has written more than 220 peer-reviewed publications and is a fellow of the American Ceramic Society, ASM International, and the American Nuclear Society (ANS). He has received numerous awards, including the U.S. Department of Energy E.O. Lawrence Award, the IEEE Nuclear and Plasma Sciences Society Fusion Technology Award, and the ANS Mishima award for outstanding R\&D on nuclear fuels and materials.

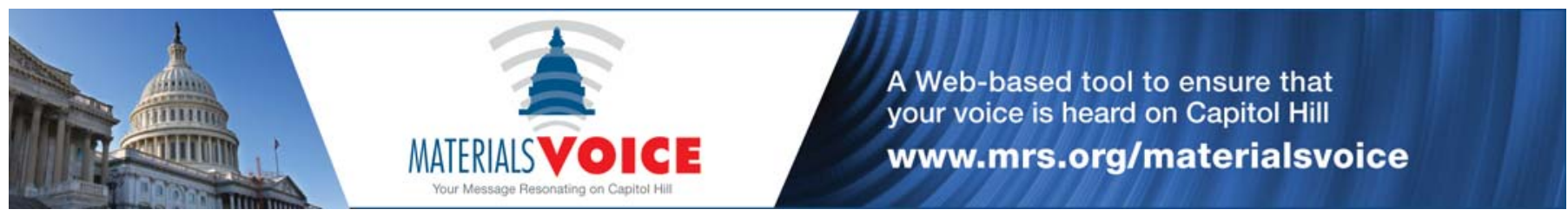




\section{PARTICLE ACCELERATOR SYSTEMS}

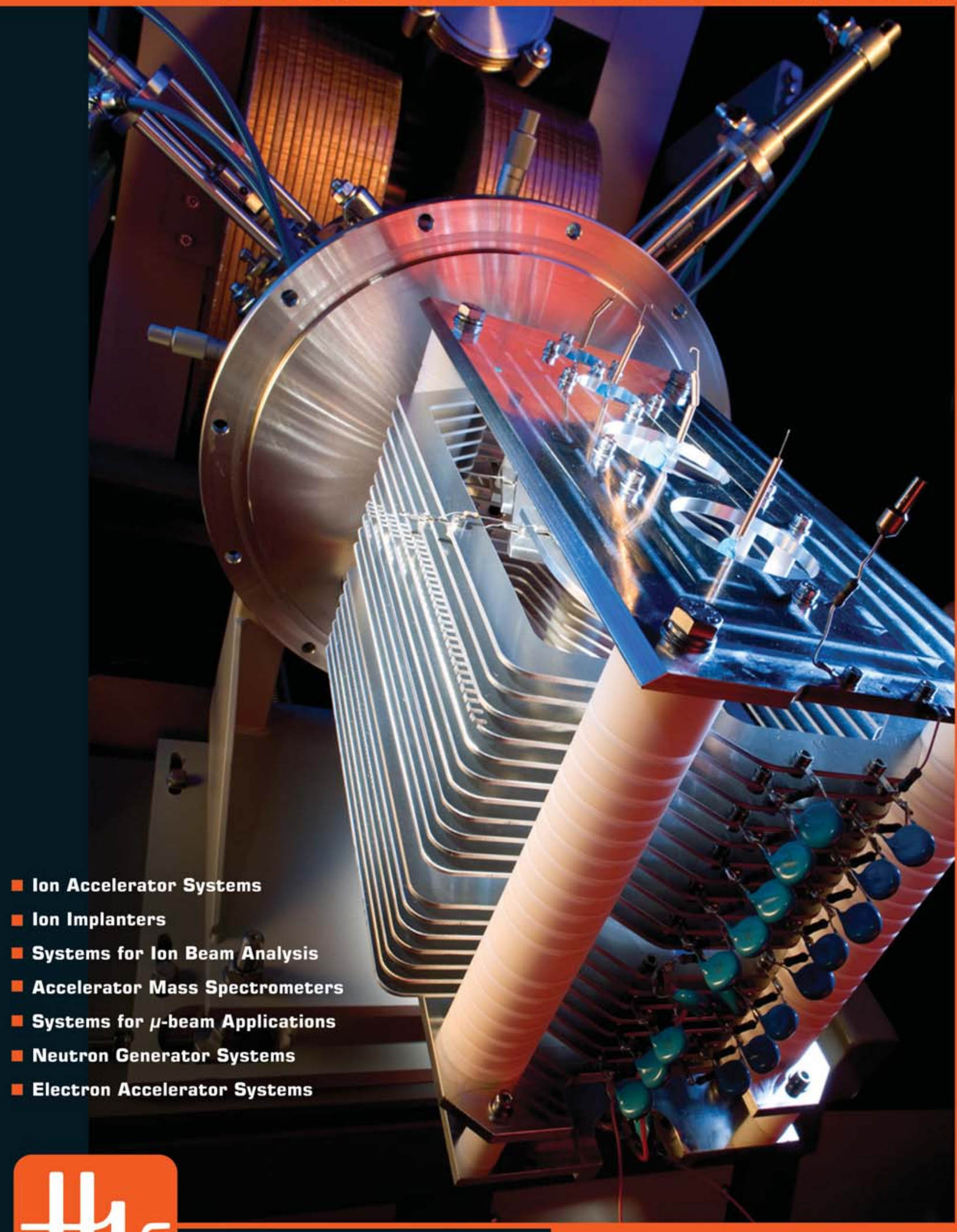

Accelerator Systems

High Voltage Engineering Europa B.V.

P.O. Box 99, 3800 AB Amersfoort, The Netherlands Tel: $31334619741 \cdot$ info@highvolteng.com www.highvolteng.com 


\section{.ل}

\section{Ultrahigh Resolution Analytical Performance}

\section{Ultralow kV}

Uncoated

stretched

polypropylene

\section{LABe}

1-3 $\mathrm{nm}$ twinning in mineral BSE image
STEM

CNT with

1-3 $\mathrm{nm} P t$

nanoparticles
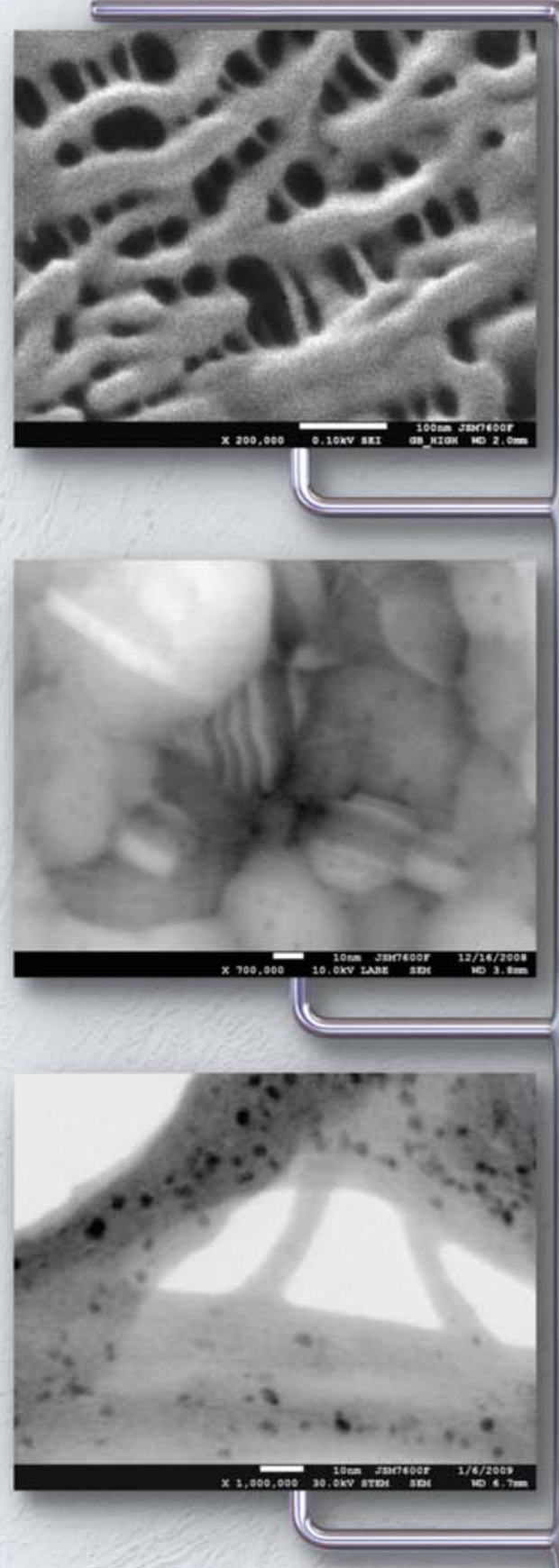

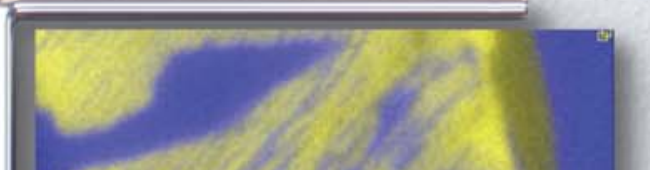

EDS/WDS

EDS map of

$\mathrm{TiO}_{2}$ in $\mathrm{FeOX}$

with $<100 \mathrm{~nm}$

spatial resolution $30,000 x$

\section{EBSD}

Orientation

map of Ni alloy

CL

Quartz and

zinc oxide

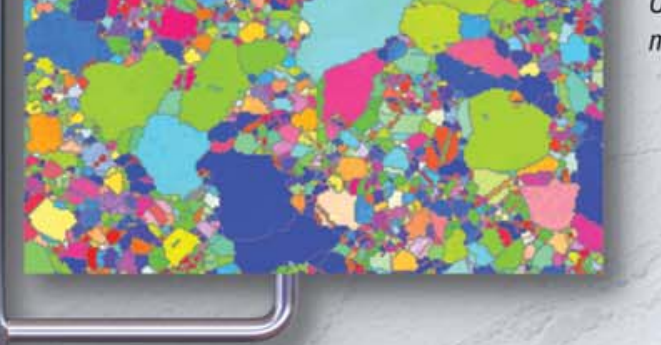

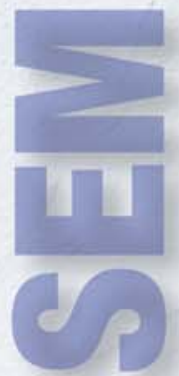
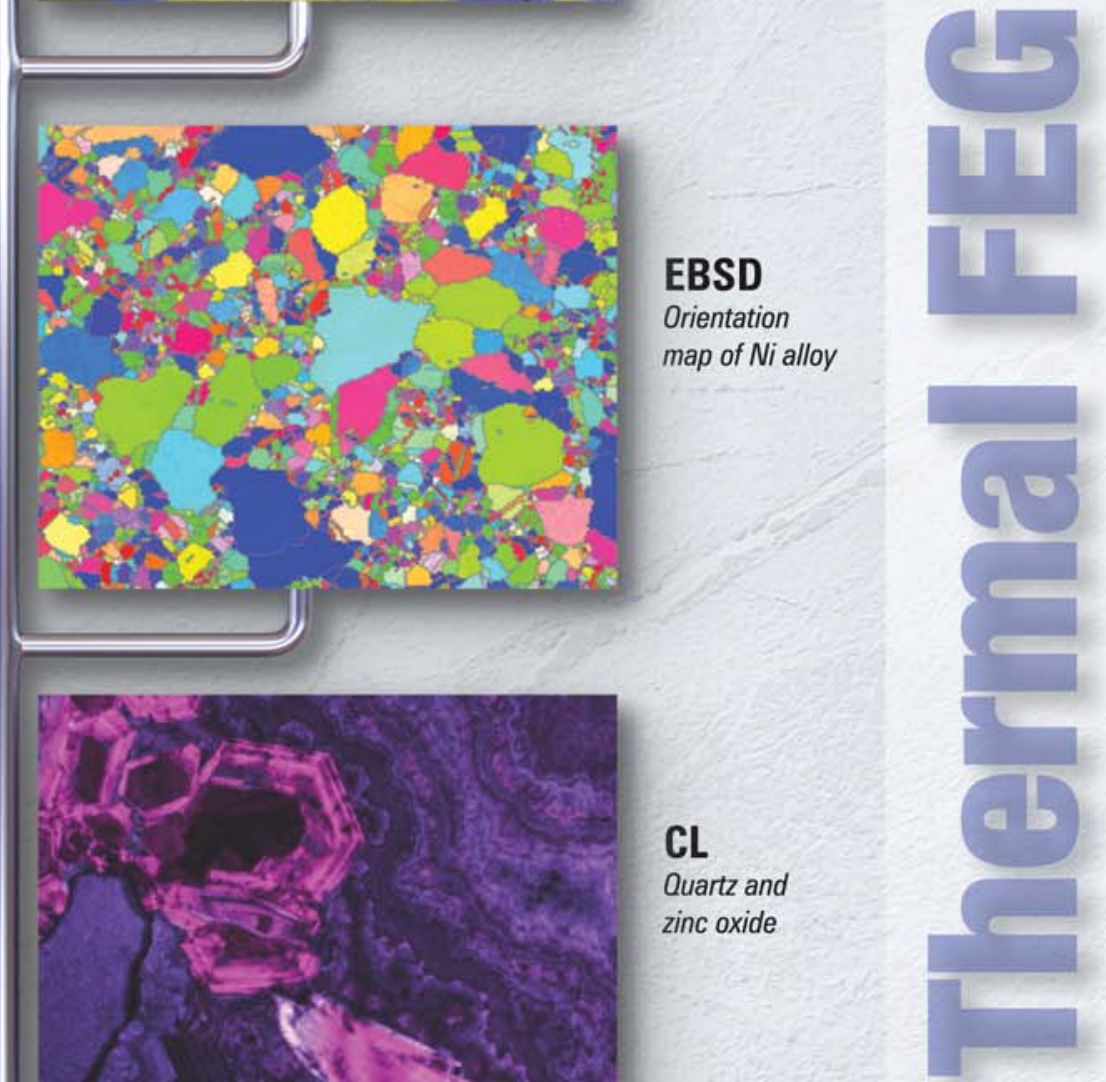

\section{Seeing is believing!}

Contact salesinfo@jeol.com to arrange a demo. For more information or to view a brochure visit www.jeolusa.com/7600F.

All magnifications as printed at $4 \times 5$ in

\section{(1)}
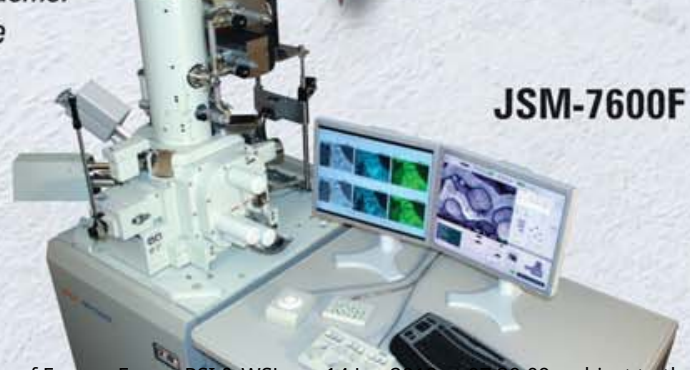